\title{
Towards a Better Future for Canadians with Bipolar Disorder: Principles and Implementation of a Community-Based Participatory Research Model
}

\author{
Erin E. Michalak, Kathleen Lane, Rachelle Hole, Steven J. Barnes, Nasreen Khatri, \\ Sara Lapsley, Victoria Maxwell, Roumen Milev, Sagar V. Parikh, Lesley Berk, \\ Michael Berk, Samson Tse, Greg Murray, Guillermo Perez Algorta, Fiona Lobban, \\ Steven Jones, and Sheri L. Johnson
}

\begin{abstract}
The Collaborative RESearch Team to study psychosocial factors in bipolar disorder (CREST.BD) is a multidisciplinary network dedicated to advancing science and practice around psychosocial issues associated with bipolar disorder (BD), improving the care and wellness of people living with bipolar disorder, and strengthening services and supports for these individuals. CREST.BD specializes in community-based participatory research, in which research is conducted as a partnership between researchers and community members. This article describes the evolution of the CREST.BD network and CREST.BD's commitment to community-based participatory research in bipolar disorder research. Examples of CREST.BD projects using community-based participatory research to study stigma, quality of life, psychosocial interventions, and creativity in bipolar disorder are highlighted, and opportunities and challenges of engaging in communitybased participatory research in bipolar disorder specifically and the mental health field more broadly are discussed. This article demonstrates how CBPR can be used to enhance the relevance of research practices and products through community engagement, and how community-based participatory research can enrich knowledge exchange and mobilization.
\end{abstract}

KEY Words bipolar disorder, community-based participatory research, community engagement, CREST.BD

\section{Introduction}

Bipolar disorder (BD), a mood disorder affecting about half a million Canadians, can be associated with profound impairments in health and quality of life (QoL) and has significant associated healthcare costs. Yet with optimal support, care and empowerment, people with bipolar disorder can flourish, leading healthy, fulfilling lives, and making creative, innovative and important contributions to society (Suto, Murray et al., 2010; Murray, Suto et al., 2011).

Research into bipolar disorder has expanded rapidly over the past decade. Much of this

Engaged Scholar Journal: Community-Engaged Research, Teaching and Learning 


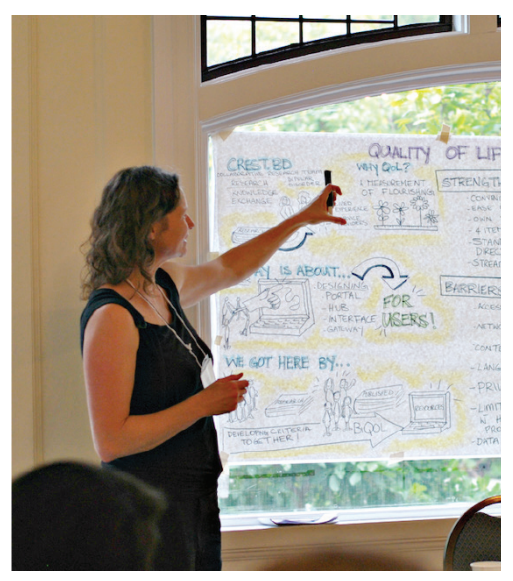

Graphic facilitation in action at a CREST.BD's Community Engagement Event to develop a new online ehealth tool. (Photo: Nusha Balram)

research has been conducted from a biomedical perspective, examining the biological causes and consequences of the condition, and pharmacological approaches to treatment. Notwithstanding the importance of this work, living well with bipolar disorder requires more than pharmacology; psychosocial factors and interventions can have a powerful impact on how the condition manifests. Although research on psychosocial interventions for bipolar disorder is expanding rapidly (Geddes and Miklowitz, 2013), significant gaps remain in understanding the influence of psychosocial factors that do not fit a traditional biomedical model (e.g., stigma, spirituality, and social support) on outcomes for people with bipolar disorder.

Community-based participatory research (CBPR) is a partnership-based philosophy of research that has been applied successfully to other health conditions to identify optimal ways of translating research findings into real-world improvements in health and wellbeing. CBPR has been particularly successful in tackling complex issues affecting health and wellbeing that do not fit well within a biomedical model. The aim of this article is to describe the evolution of a unique Canadabased network that specializes in the application of CBPR in bipolar disorder research and knowledge exchange (KE).

\section{Community-Based Participatory Research}

CBPR has been defined as research that is conducted as an equitable partnership among researchers, practitioners, and community members living with a particular health condition, disability or issue (Israel et al., 2010; Israel et al., 1998). It is characterized by substantial community engagement in all stages of the research process, from formulating study goals and hypotheses, to planning the sampling, design, measures and analyses, to disseminating results. Community encompasses patients or "users" of mental health services, people who are not receiving medical care but have lived experience of the disorder, and people within the social support network of the affected individual, including family members, caregivers, significant others, and healthcare providers. Preferred terms for those affected by bipolar disorder symptoms vary substantially (Hollander, 2011, p.456; Shaw, 2012), but we use the terms "consumer," "user," and "people with lived experience" interchangeably here. Regardless of terminology, the goal

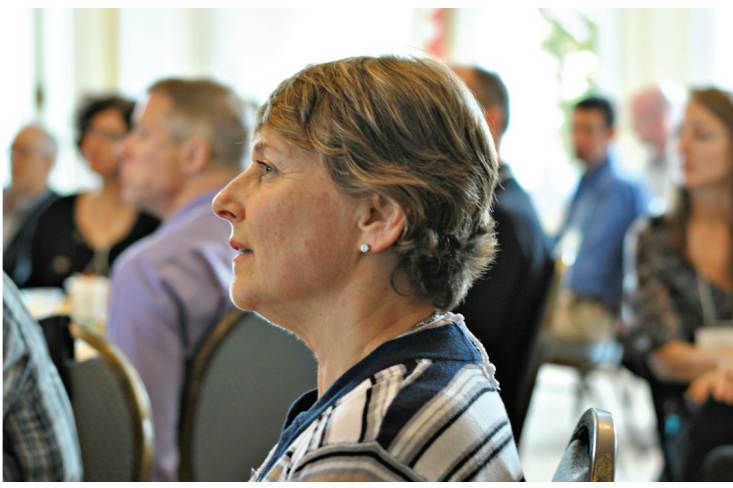

Sophia Van Norden, co-chair of the CREST.BD Community Advisory Group, 2013 Quality of Life Summit (Photo: Nusha Balram) 
of CBPR is to shape the research process to fit the perspectives of community members so as to generate knowledge that contributes more directly to social change, rather than perpetuating the notion of community members as passive objects of research (Cargo \& Mercer, 2008; Michalak et al., 2012; Wallerstein \& Duran, 2006). As a consequence of the focus on community engagement and the rich array of research questions raised by the diversity of participants, openness to adopting diverse methods is prized (Israel et al., 2005; Israel et al., 1998). CBRP approaches may encompass the flexible use of quantitative, qualitative, mixedmethod and arts-based approaches (Minkler, 2005). In turn, the use of these diverse methods draws on the integration of stakeholders' diverse strengths and expertise, including researchers' theoretical and methodological skills, practitioners' practice knowledge and experience, and family members' and consumers' lived experience expertise (Cargo \& Mercer, 2008).

A key CBPR emphasis is on "the participation and influence of non-academic researchers in the process of creating knowledge," including consumers, family members, and practitioners (Israel et al., 1998, p. 177). Some of the enduring principles identified by CBPR pioneers include: 1) recognizing the community as a unit of identity in the CBPR process; 2) building on the strengths and resources within the community; 3) facilitating collaborative partnerships in all phases of the research; 4) integrating knowledge and action for mutual benefit of all partners; 5) promoting a co-learning and empowering process that attends to social inequalities; 6) involving a cyclical and iterative process; 7) addressing health from both positive and ecological perspectives; and 8) disseminating findings and knowledge gained to all partners (Israel et al., 1998). Taken together, all eight principles underscore the importance of community throughout CBPR projects.

\section{The History of CREST.BD}

The Collaborative RESearch Team to study psychosocial factors in bipolar disorder (CREST. BD) was established in 2007 with an overarching mission of advancing psychosocial research and knowledge exchange in bipolar disorder, with the aim of improving health and quality of life for people living with bipolar disorder and strengthening services and supports for these individuals. A one-year team planning grant was secured from the British Columbian Michael Smith Foundation for Health Research. With seed funding in place, a team of founding members was formed including multidisciplinary researchers with expertise in the psychosocial aspects of bipolar disorder, healthcare providers, and people with lived experience of bipolar disorder. Of course, CREST.BD team members may hold more than one stakeholder/member role: e.g., person with lived experience/family member and academic researcher, practitioner and academic researcher, etc.; as such they may be part of both CREST.BD and the bipolar community. Such a reality highlights that personal roles within groups and communities can be multiple and overlapping.

For the first several years, CREST.BD was without core infrastructure funding. Small grants, modest environmental support, and goodwill from team members enabled the team to host annual community engagement days and continuing education events for professionals, and to recruit trainees, peer-researchers and others to join its community consultation group. In 2009,

Engaged Scholar Journal: Community-Engaged Research, Teaching and Learning 
these efforts were rewarded when CREST.BD's network grant application was ranked first in the Canadian Institutes of Health Research (CIHR) 'Knowledge to Action' competition. The resulting $\$ 200,000$ grant enabled the network to establish components necessary for growth, including hiring a knowledge exchange manager and establishing a community advisory group. In 2011, CREST.BD received close to $\$ 600,000$ in funding from the inaugural CIHR Network Catalyst competition to establish a national network. The retention of the knowledge exchange manager and the hiring of a network manager played a critical role in supporting the development and implementation of a strategic plan for the network.

CREST.BD aims to implement the key CBPR principles outlined above. Of greatest importance, CREST.BD values CBPR across all research stages, from identifying research priorities to disseminating research insights into real-world practice. That said, the extent to which any one or a combination of the eight principles can be implemented in research varies depending on the context, purpose and participants involved; the principles represent aspirational goals (Israel et al., 1998, p. 177-178). Indeed, as has been acknowledged elsewhere (e.g., Cargo \& Mercer, 2008), "many academic researchers and their partners struggle with how to operationalize participatory research principles, steps and guidelines."

Several principles have been offered to evaluate whether teams are successfully implementing CBPR (Cargo \& Mercer, 2008). Given that there is considerable variability across teams in how CBPR is applied, we consider how some of these dimensions have shaped CREST.BD.

Cargo \& Mercer (2008) identified a core set of CBPR values, including translating knowledge into action (utilization), social and environmental justice, and self-determination. These values guide CREST.BD's governance structures. These values also shape the way that CREST.BD's research programs (in the target areas of stigma, quality of life (QoL), and psychosocial interventions) developed - each was nominated and then chosen as a priority through iterative community consultation and engagement activities. This strong engagement of community members enhances the relevance and importance of the research for individuals with experiences of bipolar disorder and healthcare providers (Minkler, 2005; Plumb et al., 2004). In this way, CBPR produces scientific knowledge in ways that are most meaningful and relevant to those people most affected by the issue being studied. All three research priorities contribute to social and environmental justice and self-determination for individuals who experience bipolar disorder and their families.

A second core facet of CBPR is engaging community members in the research process. To facilitate this, the Community Advisory Group for CREST.BD consists of approximately 10 members representing people living with bipolar disorder, BD healthcare providers, and partner organization representatives. This group provides feedback and guidance across CREST. BD's ongoing research and knowledge exchange activities. More specifically, the Community Advisory Group: 1) Acts as a resource to CREST.BD in terms of planning, implementation, distribution and evaluation of research studies and knowledge exchange; 2) Helps to generate solutions to barriers within the research and knowledge exchange initiatives; 3) Plays a key role in optimizing networking opportunities with the wider BD community; 4) Functions as a communications vehicle to the BD community on the work and plans of CREST.BD; and 
5) Problem-solves barriers and solutions within the team's research and knowledge exchange initiatives (Michalak et al., 2012, p. 6).

A third core facet of CBPR is building capacity for community engagement through strategic partnership building. To further enhance the reach of its community engagement, CREST.BD has established effective partnerships with approximately 17 diverse community and clinical organizations (e.g., Canadian Network for Mood and Anxiety Treatments (CANMAT), Mood Disorders Association of Ontario (MDAO), and a 500+ person national community consultation group). The partnership with CANMAT, in particular, increased collaboration with senior clinicians, created a conduit for enhanced research partnerships, and allowed for use of CANMAT's well-established expertise and credibility in knowledge exchange and continuing professional development activities for healthcare providers. The partnership with MDAO, the largest Canadian community mood disorder organization, supported collaborations with people living with bipolar disorder and their family members, via the organization's 54 peer support groups and contact with 26,000 people affected by mood disorders annually. These partnerships provide access to cross-disciplinary, complementary expertise.

A fourth principle of CBPR is a thoughtful and equitable approach to defining how stakeholders participate. Rather than assume that all stakeholders provide equal contributions, the focus is on equitable engagement, reflecting thoughtful consideration of appropriate, democratic and informed decisions. As Cargo and Mercer (2008) observe, "How much and in what phases academic and non-academic partners should participate depends on where the interests, expertise, and energy of the partners reside; what is negotiated; and the extent to which partnership and project governance structures have made provisions to support the agreed on participation level" (p. 332). CREST.BD has a rich history of involving stakeholders as Community Advisory Group members, and in every step of the research process and knowledge exchange, as we will describe below.

\section{Examples of Community-Based Participatory Research in CREST.BD Research}

The CREST.BD vision is "a world where people living with bipolar disorder enjoy optimized health and quality of life and minimal stigma across their lifespan as a result of tailored psychosocial and/or self-management interventions informed by effective psychosocial research and KE in BD." The vision is supported by prioritizing research on stigma, quality of life, and psychosocial interventions through five strategic priorities: research and knowledge exchange excellence, community involvement, capacity building, international engagement and recognition, and sustainability. The following sections describe how CREST.BD applies CBPR in research on stigma, quality of life, psychosocial interventions, and creativity and bipolar disorder.

\section{Stigma}

In response to feedback from the community, CREST.BD initiated a program of research into stigma in bipolar disorder (Michalak et al., 2012). Stigma concerning mental illness is a serious concern for people with bipolar disorder and their caregivers (Hawke, Parikh \& Michalak, 2013)

Engaged Scholar Journal: Community-Engaged Research, Teaching and Learning 
and leads some individuals to avoid or discontinue mental health treatment (e.g. Livingston \& Boyd, 2010). First, a community engagement day and a continuing professional development event focused on stigma and bipolar disorder were held. A qualitative research study using focus groups was embedded into the community engagement day to examine definitions, experiences and subjective impact of stigma as well as to discuss possible stigma reduction interventions. People with lived experience of bipolar disorder collaborated on the design, implementation and analysis of both the community engagement day and the qualitative research project (for example, developing focus group questions and methods, conducting focus groups, analysing focus group data) and were co-authors of the resulting peer-reviewed publication (Suto, Livingston, Hole et al., 2012).

Findings from the event, together with a literature review that highlighted the potential impact of stigma among healthcare providers, were used to secure funding for a project to examine whether a purpose-built theatrical performance could help reduce stigma. Co-investigators on the grant included people from multiple fields of expertise: lived experience, psychology, psychiatry, and social work. One of the grant co-investigators (Victoria Maxwell), an actress and mental health educator with lived experience of bipolar disorder, produced and performed a one-hour, one-woman theatrical performance entitled 'That's Just Crazy Talk' in which the narrator described her personal and familial mental illness, her and her family's experiences of mental health stigma, and attempts to come to terms with a complex illness. The CREST.BD community advisory group and network partners (for example, CANMAT, MDAO) supported the development, implementation and evaluation of the performance. Findings revealed that the performance significantly reduced stigma among healthcare providers as measured quantitatively (Michalak et al., 2014), and among both people with bipolar disorder and healthcare providers as measured qualitatively (Michalak et al., 2014). A filmed version of the performance was found to diminish negative attitudes in healthcare providers (Hawke et al., 2014). The live and filmed versions of 'That's Just Crazy Talk' have been disseminated widely, with its broad uptake echoing the commitment to multiple stakeholders as exemplified by the research team composition. In its live form, the performance has now been seen by over 7000 people in North America, including performances at major international medical conferences, post-secondary institutions and community organizations. The intervention is now fully integrated into on-going presentations by Victoria Maxwell. More than 500 copies of the filmed version of the performance are now in circulation, and it has been adopted into official curricula by post-secondary nursing programs (e.g. Queen's University), professional bodies (e.g. National Society of Genetic Counselors) and the Mental Health Commission of Canada's Opening Minds program — the largest systematic effort in Canadian history focused on reducing mental illness stigma.

\section{Quality of Life}

With a strong influence from biomedically-focused disease models, mood symptoms have long been a primary outcome measure within bipolar disorder research (Zachar \& Kendler, 2007). People with lived experience, however, may weight recovery and quality of life (Jones, Mulligan, Higginson, Dunn \& Morrison, 2012; Murray \& Michalak, 2012; Maxwell \& Michalak, 
2011; Michalak et al, 2012; Tse et al, 2013) as more important than symptom relief. In bipolar disorder, symptoms account for just a proportion of the variance in quality of life outcomes.

In our second example of CBPR, people with bipolar disorder were involved in all stages of developing the first bipolar-specific quality of life scale, with content that covers subjective meaning along with traditional domains of functioning (Michalak \& Murray, 2010). The Quality of Life in bipolar disorder (QoL.BD) scale has become the gold-standard for BD-specific quality of life assessments, as illustrated by its translation into more than 19 languages, and use in over 16 large-scale clinical studies. Implementation of the scale in practice was aided substantially by diverse community-engagement methods. For example, people with lived experience of bipolar disorder supported the design and delivery of in-person training workshops and online training videos for healthcare providers, in-person and online (webinar) presentations, QoL-focused outputs for the CREST.BD research blog, and social media (Facebook, Twitter) outputs. As one concrete example, the CREST.BD network lead and Victoria Maxwell provided the keynote talk, a lived-experience perspective talk, and a workshop, at the 2015 Calgary Mood Day conference, which was geared for BD healthcare providers. Network lead Michalak first presented on results from CREST.BD's program of research into quality of life, and on the philosophy of CBPR in $\mathrm{BD}$ research and KE; Victoria Maxwell then provided readings on lived-experience perspectives of quality of life. Then they co-presented a workshop on pragmatic tools for integrating quality of life assessments into routine clinical care, the content of which was generated by prior CREST. BD research into quality of life conducted within a CBPR framework.

To foster use of the QoL.BD in healthcare and personal health management, CREST.BD developed a web-based version of the QoL.BD - the QoL Tool. Funding for the project came in the form of a CIHR "ehealth Catalyst" grant; as in all of CREST.BD's funding, people with bipolar disorder were named co-investigators, primary decision makers or knowledge users on the funding application. The QoL Tool itself was developed hand-in-hand with the $\mathrm{BD}$ community. For example, for this project, a community engagement day was initially held in Vancouver to consult on the design features and appearance of the QoL Tool. Graphic facilitation was incorporated into the day, the outputs of which were used to support the development of an online presentation narrated by a person with bipolar disorder. Team members also created social media outputs and a series of research blogs. The resulting QoL Tool provides interactive results to chart quality of life over time. Live and on-line training for clinicians was offered to facilitate the scale's adoption among healthcare providers.

\section{Psychosocial Interventions}

Effective self-management strategies are important for empowering people with bipolar disorder and contribute to improved health outcomes and quality of life (Murray et al, 2011). To foster better knowledge of effective self-management strategies for bipolar disorder, CREST.BD drew on the expertise of participants who employed effective self-management strategies and identified as "living well" with bipolar disorder (Suto, Murray, Hale, Amari, \& Michalak, 2010). CREST.BD then collaborated with peer researchers to conduct an extensive (academic and grey) literature search, and then a large sample of community members and healthcare practitioners 
were asked to rate self-management strategies in a series of online surveys (Michalak et al, 2013).

To maximize access to the findings regarding self-management, CREST.BD has developed an interactive website (the Bipolar Wellness Centre) that incorporates the online QoL Tool to provide users with evidence-based self-management strategies tailored to their personal quality of life profile. Several approaches to optimal knowledge exchange through the Bipolar Wellness Centre

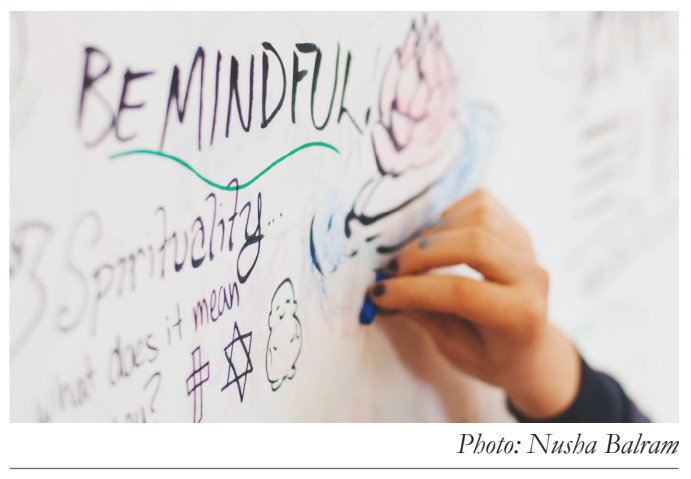
are currently being evaluated. For example, in the Living Library option, people with bipolar disorder or healthcare providers borrow a trained peer and engage in short training sessions (via live and secure web technology) to learn to analyze QoL assessment results, use the Centre to examine QoL strengths and deficits, and apply targeted self-management strategies. Other implementation approaches include the production of 14 QoL-domain specific webinars (produced either by academic or lived experience network members), a travelling roadshow, in which a series of workshops are delivered by both people with bipolar disorder and academic researchers, and the production of 6 videos that show concrete examples of self-management in action in a person with lived experience of bipolar disorder.

\section{Creativity}

Of the various positive features of bipolar disorder, creativity is perhaps the most frequently mentioned advantage, and CREST.BD has used CBPR to understand the elevated levels of creativity observed among people with bipolar disorder. Funding from CIHR provided support for hosting a community engagement day attended by people with bipolar disorder working as musicians, artists, authors, or other creative professions. As part of the day, focus groups explored participants' perceptions of the mechanisms linking bipolar disorder and creativity. Although the literature in this area has tended to focus on a small number of potential mechanisms, such as divergent thinking, energy, or ambition, the affected individuals suggested a much broader and more individualized range of potential mechanisms, ranging from the ability to use rich life experiences as a base for novels, the use of artistic pursuits for political or emotional expression, and the flexibility of work schedules afforded to those with creative pursuits (Johnson et al, in Press). This community input helped shape new hypotheses for research on creativity, and highlighted the importance of using the creative strengths of those with bipolar disorder for promoting treatment engagement and reducing stigma.

\section{Strengths, Challenges and Opportunities of Community-Based Participatory Research in Bipolar Disorder}

\section{Strengths}

We believe that the four example projects described above demonstrate the value of CBPR 
in $\mathrm{BD}$ research. In each project, the interpretation of research outcomes was enriched by the integration of different perspectives, as was dissemination (Cargo \& Mercer, 2008).

From a process perspective, CBPR naturally facilitates KE because research is conducted within the community in which knowledge will be applied (McGrath, Lingley-Pottie, Emberly, Thurston \& McLean, 2009). CBPR may also increase empathy in both directions, as healthcare providers learn to appreciate the challenges of living with bipolar disorder, and community members see the healthcare providers in a broader context, appreciating the demands on them and the constraints of the system within which they work.

It is also important to note a strength of CBPR that has emerged from research conducted at a wide range of sites: a focus on understanding positive features of bipolar disorder (Murray \& Johnson, 2010; Seal, Mansell \& Mannion, 2008). In surveys, those with lived experience value their heightened emotional sensitivity, alertness, productivity, social engagement, sexual enjoyment, creativity, spirituality, empathy, realism, and resilience as correlates of the disorder (Galvez, Thommi \& Ghaemi, 2011). In a study in which a researcher with lived experience of bipolar disorder conducted interviews and analyzed the data, findings highlighted the value of bipolar disorder in amplifying certain cognitive abilities and promoting a sense of human connectedness (Lobban, Taylor, Murray \& Jones, 2012).

\section{Challenges}

As with any approach to research, there are specific challenges in conducting CBPR, especially in the mental health domain. Much of the discussion below, speaking to priorities, people and policies, has implications for informing decisions about what participation in CBPR might look like.

Priorities. Our experience has been that people with bipolar disorder and their family members place high value on promoting recovery and advocating for social change; researchers value the team's research activities. Whilst the two goals are not mutually exclusive, they are not always fully integrated; CREST.BD's primary funding remit is research and knowledge exchange, and at times strategic leadership has been required to ensure there is not undue drift away from this core mission. Having said, this, we have identified a number of strategies effective for meeting diverse objectives and goals in our work. For example, we rarely hold a community engagement event that does not include a formal research component (for example, focus groups, qualitative interviews, quantitative surveys). Even informal community engagement activities (for example, structured online Tweetchats around an area of interest in BD research and knowledge exchange) are viewed as an opportunity to obtain potential pilot 'data' for future funding applications or as a mechanism to support the identification of new avenues of research. At the same time, we strive to create space in each event for participants with lived experience to network, advocate, tackle stigma, become more empowered and work towards personal recovery.

People. As in any group of people, some individuals with lived experience are more willing and able to collaborate than others (Wallerstein \& Duran, 2006). Notwithstanding the multiple roles of CREST.BD members and partners in relation to bipolar disorder, we agreed it could be

Engaged Scholar Journal: Community-Engaged Research, Teaching and Learning 
prudent to focus efforts initially on developing deep relationships with a small number of peer researchers. On the other hand, the most disadvantaged and difficult to engage members of the community may benefit the most from CBPR, and also have some of the most important contributions to make. Thoughtful engagement strategies, appropriate training, and ongoing evaluation are being applied as the network develops and expands in order to address barriers to participation from more marginalized or hard-to-reach communities.

Policies. CBPR can play a major role in policy changes, but this does not happen automatically. Research is most likely to influence policy if the policy makers are brought into the dialog early to shape the types of data that they will need, and then remain engaged throughout critical steps in the process. Although many researchers feel somewhat uncomfortable with the difficulties of translating research into other realms, researchers who consider policy implications as a core part of their role may be more effective over time in ensuring that their findings have meaning for the community. In other areas, CBPR has skillfully included politicians and policy makers in the research planning process (Wallerstein \& Duran, 2006), which has not only allowed researchers to refine their data collection to address critical needs in public planning, but also allowed for rapid dissemination of findings into the hands of policy makers.

\section{Opportunities}

CBPR on the Web. Another potential area for growth in CBPR is through the use of online communities that harness modern web technologies, such as those that permit users to contribute and debate online (see Brossard \& Scheufele, 2013; Stellefson et al., 2013). Thus far, CREST.BD has had considerable success in employing web technologies to build social networks and visibility and thereby enhance our CBPR and knowledge exchange activities (McBride et al., in Press). Modern web technologies that can enhance knowledge exchange and open new avenues of research are part of the emerging area of e-health in mood disorders (Parikh \& Huniewicz, 2015). For example, Twitter is a public messaging and social networking platform with massive following. A tool like Twitter could be used in CBPR to explore new research avenues (e.g., Peace \& Myers, 2012). For a person living with a mental illness in a remote region, being part of an online community of like-minded individuals can be an empowering source of support. Input from individuals with bipolar disorder who are unreachable by traditional research methods might reveal as yet unknown relationships between environmental and social variables and the incidence or presentation of bipolar disorder. Moreover, ongoing real-time analyses of social networks will be a rich way of yielding new insights into the needs of the BD community (see Gruzd \& Haythornthwaite, 2013). In pursuing these agendas, the relationship between web technologies and CBPR should not be thought of as a one-way street. To ensure maximal community involvement, CBPR should be used as a means of supporting iterative user-centered design (Marriott et al., 2012) of new internet-based tools for the BD community (Henderson et al., 2013).

Funding. Some funding agencies directly support CBPR; however, traditional funding structures impose a range of challenges to achieving the CBPR ideal of involving community members throughout all research phases. Research teams must fund the grant development 
process, which may limit opportunities for involvement of multiple stakeholders during hypothesis generation, discussion, and research design. Funding agencies may also not build in flexibility in adapting to periods when peer researchers with lived experience are less able to contribute due to a period of illness. In the early years of CREST.BD's evolution, small awards of alternative funding (such as CIHR meeting and dissemination grants and funding from individual members' own institutions) proved critical for supporting ongoing community engagement in the gaps between project-specific operating grants. The newly launched CIHR Foundation Scheme, designed to provide long-term programmatic support for innovative research, offers an exciting alternative funding mechanism that is ideally suited to CBPR. These changes to funding practices in the Canadian healthcare funding realm may offer significant opportunities for community-engaged scholars to successfully and authentically implement action orientated research in the future.

\section{Conclusions and Future Directions}

Increasingly CBPR is being used to address complex public health and mental health issues because of its potential for bridging gaps between research and practice (Cargo \& Mercer, 2008); nonetheless, it is a relatively nascent practice in BD research. Given the many strengths of CBPR — including the inherent flexibility of research methods used (e.g., quantitative, qualitative, mixed-methods), the engagement with and buy-in of stakeholders that can enhance knowledge production around issues of relevance to the community, and the positive implications for knowledge exchange and knowledge mobilization CREST.BD has found that that CBPR is a complementary and value-added approach to traditional research practices.

Although CBPR can challenge traditional research paradigms, it is clear that when research is connected to and conducted with the communities who have a stake in the issue under study, it will enhance the value of research for those communities (Hacker, 2013; Minkler 2005). Further, given the emphasis on action, CBPR can help address the gulf that often occurs in mental health research between the knowledge that research produces and its application (Israel et al., 1998). It is important to underscore the increasing funding opportunities available to researchers engaged in CBPR both in Canada and internationally. While there remains criticism of CBPR's production of localized knowledge for specific actions in a particular community, there is increasing validation from funders that CBPR produces worthwhile applied knowledge and produces action to address complex social issues.

In conclusion, using examples of CBPR in practice, this article demonstrates strengths, challenges and opportunities when employing CBPR in BD research. While there are challenges in conducting CBPR, engaging community stakeholders offers great opportunities for meaningful research. Finally, we echo the call of Roche (2008) for a critical examination and attentiveness to the practices that shape CBPR as well as improved strategies for evaluating the impact and outcomes of action resulting from CBPR. 


\section{About the Authors}

Guillermo Perez Algorta obtained a PhD in clinical psychology at the Universidad de Palermo of Buenos Aires, Argentine, in 2009. Currently, he is a lecturer in mental health at Lancaster University, UK. He was also a postdoctoral researcher in the Department of Psychiatry at Ohio State University (2012 - 2014).

Steven Barnes is co-deputy lead of the CREST.BD network, a faculty member in UBC's Department of Psychology, and has lived experience with BD. His neuroscientific expertise lies in the areas of psychiatric disorders, epilepsy, learning and memory, and neuroplasticity. He is co-author of the forthcoming 10th edition of a best-selling textbook on Behavioural Neuroscience, Biopsychology (Pearson).

Lesley Berk is an Alfred Deakin Postdoctoral Research Fellow in the School of Psychology at Deakin University in Australia. Currently she is developing and evaluating online information resources and psychosocial interventions to assist family and friends to better understand and deal with the bipolar disorder or depression of a loved one, and end-users of these interventions are actively involved in this process.

Michael Berk is a NHMRC Senior Principal Research Fellow and Alfred Deakin Professor of Psychiatry at Deakin University. His interests are the development and implementation of novel therapies.

Rachelle D. Hole, PhD, is an associate professor in the School of Social Work, Faculty of Health and Social Development, co-director of the Centre for Inclusion and Citizenship and the Centre for Social, Spatial and Economic Justice at The University of British Columbia, Kelowna, British Columbia, Canada.

Sheri L. Johnson, Ph.D. is the director of the Cal Mania (CALM) Program and a professor of psychology at the University of California Berkeley. Her work has focused on understanding the triggers of mania for the past 20 years, with funding from NIMH and NARSAD.

Steven Jones received his $\mathrm{PhD}$ and clinical training at the Institute of Psychiatry in London, and is the founding Director of the Spectrum Centre for Mental Health Research. He has over 100 publications, mainly on the development of cognitive therapy approaches for bipolar disorder and on psychological models relevant to the development and recurrence of bipolar experiences.

Nasreen Khatri is a registered clinical psychologist and researcher at the Rotman Research Institute, Baycrest in Toronto. She studies how mood disorders impact the aging brain and innovates CBT treatments for older adults. 
Kathleen Lane is a consultant and project manager specializing in research to improve health and wellbeing. She is an MBA candidate at the UBC Sauder School of Business and previously studied population health at the University of British Columbia Okanagan.

Sara Lapsley, B.A., M.A. is the Counsellor and Group Clinician at the BC Forensic Psychiatric Hospital and a Peer Researcher for CREST.BD. Her interests are: Mental Health and the Law, Bipolar Disorder, and qualitative research methods.

Fiona Lobban is the Co-Director of the Spectrum Centre for Mental Health Research. Her research focuses on the development and evaluation of psychological interventions for people with bipolar disorder and related experiences, with the aim to increase access to effective psychological support for people with mental health problems and their relatives.

Victoria Maxwell is a speaker, playwright, actor and peer researcher. She tours her one person theatre shows about recovering from mental illness across Canada and the States. In addition, she is a founding member of CREST.BD, and blogs for Psychology Today.

Erin E. Michalak (corresponding author) earned her PhD at University of Wales in the United Kingdom and is currently an Associate Professor in the Department of Psychiatry at the University of British Columbia. She is the founder and leader of the 'Collaborative RESearch Team for the study of psychosocial issues in Bipolar Disorder. Email: erin.michalak@ubc.ca

Roumen Milev is a professor of Psychiatry and Psychology, and Head of department of psychiatry at Queen's University, Kingston, On, Canada. His research is in the areas of new treatments and biomarkers in Depression and Bipolar disorders and stigma of mental illness.

Greg Murray, PhD, is a Research Leader in the psychology department at Swinburne University of Technology, Australia. He is also a clinical psychologist and fellow of the Australian Psychological Society.

Sagar V. Parikh is Professor of Psychiatry at the University of Toronto, and has been newly appointed as Professor of Psychiatry, University of Michigan, where he holds an endowed chair as the John F. Greden Professor of Depression and Clinical Neuroscience, as well as Associate Director of the University of Michigan Comprehensive Depression Center.

Samson Tse is Professor of Mental Health and Associate Dean of the Faculty of Social Sciences, The University of Hong Kong. He has long standing interest in supporting individuals affected by bipolar illness and gambling disorder by using recovery approach and strengthsbased interventions. 


\section{References}

Brossard, D. \& Scheufele, D. A. (2013). Science, new media, and the public. Science, 339, 40-41.

Cargo, M. \& Mercer, S.L. (2008). The value and challenges of participatory research:

Strengthening its practice. Annual Review of Public Health, 29, 325 - 350.

Galvez, J. F., Thommi, S. \& Ghaemi, S. N. (2011). Positive aspects of mental illness: a review in bipolar disorder. Journal of Affective Disorders, 128, 185-90.

Geddes J.R., Miklowitz D.J. (2013) Treatment of bipolar disorder. Lancet, 11(381), 1672-82.

Gruzd, A. \& Haythornthwaite, C. (2013). Enabling community through social media. Journal of Medical Internet Research 15(10):e248.

Hacker, K. (2013). Community-based participatory research. Thousand Oaks, CA: Sage Publications.

Hawke, L. D., Parikh, S. V. \& Michalak, E. E. (2013). Stigma and bipolar disorder: a review of the literature. Journal of Affective Disorders, 150, 181-91.

Hawke LD, Michalak EE, Maxwell V, \& Parikh SV. (2014) Reducing stigma towards people with bipolar disorder: Impact of a filmed theatrical intervention based on a personal narrative. International Journal of Social Psychiatry, 60(8), 741-50.

Henderson, V. A., Barr. K. L. C., An, L. C., Guajardo, C., Newhouse, W., Mase, R. \& Heisler, M. (2013). Community-based participatory research and user-centered design in a diabetes medication information and decision tool. Progress in Community Health, 7, 2.

Hollander, J. B. (2011). Keeping control: the paradox of scholarly community-based research in community development. Community Development Journal, 46, 265-72.

Israel, B.A., Coombe, C.M., Cheeazum, R.R., Schulz, A.J., McGranaghan, R.J., Lichtenstein, R., Reyes, A.G., Clement, J., \& Burris, A. (2010). Community-based participatory research: A capacitybuilding approach for policy advocacy aimed at eliminating health disparities. American Journal of Public Health, 100(11), $2094-2102$.

Israel, B.A., Eng, E., Schulz, A., \& Parker, E.A. (Eds.) (2005). Methods in community-based participatory research for health. San Francisco: Jossey-Bass.

Israel, B.A., Schulz, A.J., Parker, E.A., \& Becker, A.B. (1998). Review of community-based research: Assessing partnership approaches to improve public health. Annual Review of Public Health, $19,173-202$.

Johnson, S. L., Moezpoor, M., Murray, G., Hole, R., Barnes, S. J., CREST.BD \& Michalak, E. E. (in press). Creativity and Bipolar: Igniting a Dialogue. In Qualitative Health Research.

Jones, S., Mulligan, L. D., Higginson, S., Dunn, G. \& Morrison, A. P. (2012). The bipolar recovery questionnaire: Psychometric properties of a quantitative measure of recovery experiences in bipolar disorder. Journal of Affective Disorders, 147, 34-43.

Livingston, J. D. \& Boyd, J. E. (2010). Correlates and consequences of internalized stigma for people living with mental illness: A systematic review and meta-analysis. Social Science \& Medicine, 71, 2150-61.

Lobban. F., Taylor, K., Murray, C. \& Jones, S. (2012). Bipolar Disorder is a two-edged sword: A qualitative investigation into the positive edge. Journal of Affective Disorders, 141, 204-12.

Marriott, L. K., Nelson, D. A., Allen, S., Calhoun, K., Eldredge, C. E., Kimminau, K. S., ... \& Shannon, J. (2012). Using health information technology to engage communities in health, education, and research. Science Translation Medicine, 4, $119 \mathrm{mr} 1$.

Maxwell, V, \& Michalak, E. E. (2011). Experiences and understandings of diagnosis: lived-experience perspectives. In G Murray (ed.) A Critical Introduction to DSM (7-18). NY: Nova Science Publishers. 
McBride, S., Barnes, S. J., Khatri, N., Parikh, S. V., \& Michalak, E. E. (in press). Bipolar disorder research 2.0: Web technologies for research capacity and knowledge translation. Journal of Evaluation in Clinical Practice.

McGrath, P. J., Lingley-Pottie, P., Emberly, D. J., Thurston, C., \& McLean, C. (2009). Integrated knowledge translation in mental health: family help as an example. Journal of the Canadian Academy of Child and Adolescent Psychiatry = Journal de l'Academie canadienne de psychiatrie de l'enfant et de l'adolescent, 18, 30-7.

Michalak, E. E., Hole, R., Livingston, J. D., Murray, G., Parikh, S. V., Lapsley, S. \& McBride, S. (2012). Improving care and wellness in bipolar disorder: origins, evolution and future directions of a collaborative knowledge exchange network. International Journal of Mental Health Systems, 6, $1-13$.

Michalak, E. E. \& Murray, G. (2010). Development of the QoL.BD: a disorder-specific scale to assess quality of life in bipolar disorder. Bipolar Disorders, 12, 727-40.

Michalak, E.E., Hole, R., Holmes, C., Velyvis, V., Austin, J., Pesut, B. \& Hou, S. (2012) "Recovery is such a huge word": Understandings of 'recovery' in people with Bipolar Disorder and implications for psychiatric care. Psychiatric Annals, 42(5):173-178.

Michalak, E. E., Livingston, J. D., Maxwell, V., Hole, R., Hawke, L. D. \& Parikh, S. V. (2014). Using theatre to address mental illness stigma: a knowledge translation study in bipolar disorder. International Journal of Bipolar Disorders, 2, 1.

Michalak, E.E., Suto, M., Lapsley, S.E., Scott, M., Austin, J., Berk L., \& Barnes, S.J. (2013). Delphi Consensus Consultation methods: Redefining what it means to be a bipolar expert. ISBD Global Newletter, 14 (3). Retrieved from: http://www.isbd.org/images/Volume\%2014_ Issue $\% 203 . p d f$

Minkler, M. (2005). Community-based research partnerships: Challenges and opportunities. Journal of Urban Health: Bulletin of the New York Academy of Medicine, 82 (2), Supplement 2, ii3 - ii12.

Murray, G. \& Johnson, S. L. (2010). The clinical significance of creativity in bipolar disorder. Clinical Psychology Review, 30, 721-32.

Murray, G. \& Michalak, E. E. (2012). The quality of life construct in bipolar disorder research and practice: past, present, and possible futures. Bipolar Disorders, 14, 793-6.

Murray, G., Suto, M., Hole, R., Hale, S., Amari, E., \& Michalak, E. E. (2011). Self-management strategies used by 'high functioning' individuals with bipolar disorder: From research to clinical practice. Clinical Psychology \& Psychotherapy 18, 95-109.

Parikh, S. V. \& Huniewicz, E.. (2015) E-Health: An overview of the uses of the internet, social media, apps, and websites for Mood Disorders. Current Opinion in Psychiatry, 28, 13-7.

Peace, D. M. \& Myers, E. (2012). Community-based participatory process climate change and health adaptation program for Northern First Nations and Inuit in Canada. International Journal Circumpolar Health, 71, 18412.

Roche, B. (2008). New directions in community-based research. (26 pages)Toronto, Ontario, Canada: Wellesley Institute.

Seal, K., Mansell, W. \& Mannion, H. (2008). What lies between hypomania and bipolar disorder? A qualitative analysis of 12 non-treatment-seeking people with a history of hypomanic experiences and no history of major depression. Psychology and Psychotherapy, 80, 33-53.

Shaw, I. (2012). Practice and research. Farnham: Ashgate

Stellefson, M., Chaney, B., Barry, A. E., Chavarria, E., Tennant, B., Walsh-Childers, K., Sriram, P. S. \&

Engaged Scholar Journal: Community-Engaged Research, Teaching and Learning 
Zagora, J. (2012). Web 2.0 Chronic Disease Self-Management for Older Adults: A Systematic Review. Journal Medical Internet Research, 15, e35.

Suto, M., Livingston, J.D., Hole, R., Lapsley, S., Hinshaw, S.P., Hale, S., \& Michalak, E.E. (2012). Stigma Shrinks my Bubble: A Qualitative Study of Understandings and Experiences of Stigma and Bipolar Disorder. Stigma Research and Action, 2.

Suto, M., Murray, G., Hale, S., Amari, E., \& Michalak, E. E. (2010). What works for people with bipolar disorder? Tips from the experts. Journal of Affective Disorders, 124(1-2), 76-84. doi:10.1016/j.jad.2009.11.004

Tse, S., Murray, G., Chung, K. F., Davidson, L., Ng, K. L. \& Yu, C. H. (2013) Exploring the recovery concept in bipolar disorder: a decision tree analysis of psychosocial correlates of recovery stages. Bipolar Disorders, 16, 366-377.

Zachar, P. \& Kendler, K. (2007). Psychiatric disorders: a conceptual taxonomy. American Journal of Psychiatry, 164, 557-65. 